\title{
Chronic Corticosterone Exposure during Adolescence Reduces Impulsive Action but Increases Impulsive Choice and Sensitivity to Yohimbine in Male Sprague-Dawley Rats
}

\author{
Mary M Torregrossa', Maylene Xie' and Jane R Taylor*, ${ }^{*}, 2$ \\ 'Department of Psychiatry, Yale University, New Haven, CT, USA; ${ }^{2}$ Department of Psychology, Yale University, New Haven, CT, USA
}

\begin{abstract}
Chronic stress during adolescence is associated with an increased risk for alcoholism and addictive disorders. Addiction is also associated with increased impulsivity, and stress during adolescence could alter cortical circuits responsible for response inhibition. Therefore, the present study determined the effect of chronic exposure to the stress hormone corticosterone (CORT) during adolescence on tests of impulsivity in adulthood and examined possible biochemical mechanisms. Male Sprague-Dawley rats were exposed to CORT by their drinking water during adolescence (post-natal day 30-50). The rats were then tested in adulthood to assess behavior on the 5-choice serial reaction time task (5CSRTT), stop-signal reaction time task (SSRTT), and the delay-discounting task, which differentially assess attention, impulsive action, and impulsive choice. Yohimbine-induced impulsivity on the 5CSRTT and biochemical analysis of the lateral orbital frontal cortex (IOFC) was also assessed owing to the ability of yohimbine to activate the hypothalamic-pituitary-adrenal axis and influence impulsivity. Adolescent CORT-treated rats were found to behave largely like controls on the 5CSRTT, but did show reduced premature responses when the intertrial interval was increased. Nevertheless, the CORT-treated rats tended to have more yohimbineinduced impulsive responses at low doses on this task, which was not found to be due to increased pCREB in the IOFC, but could be related to a higher expression/activity of the AMPA receptor subunit GluRI. Adolescent CORT-treated rats performed more accurately on the SSRTT, but showed greater impulsivity on the delay-discounting task, as indicated by steeper discounting functions. Therefore, adolescent CORT exposure reduced impulsive action but increased impulsive choice, indicating that chronic stress hormone exposure in adolescence can have long-term consequences on behavior.
\end{abstract}

Neuropsychopharmacology (2012) 37, 1656-1670; doi:I0.1038/npp.20 I2. I ; published online I5 February 2012

Keywords: impulsivity; adolescence; stress; yohimbine; delay discounting; lateral orbital frontal cortex

\section{INTRODUCTION}

Adolescence is a critical period for physical, social, and emotional development. Underlying many of these outward signs of development are ongoing changes in brain structure and function. In particular, stress responsive brain regions, including the prefrontal cortex (PFC), limbic system, and hypothalamic-pituitary-adrenal (HPA) axis, undergo significant developmental changes during adolescence (Lewis, 1997; Spear, 2000). In addition, stress and adverse events in adolescence are reported to increase the risk for psychiatric disorders in adulthood (Covault et al, 2007; Fox et al, 2010; Laucht et al, 2009a, b). An individual's propensity to engage in impulsive behavior or make

*Correspondence: Dr JR Taylor, Department of Psychiatry, Yale University School of Medicine, Division of Molecular Psychiatry, S307, Connecticut Mental Health Center, Ribicoff Research Laboratories, 34 Park St., New Haven, CT 06508, USA, Tel: + I 203974 7727, Fax: + I 203974 7724, E-mail: jane.taylor@yale.edu

Received 16 August 2011; revised 10 January 2012; accepted II January 2012 impulsive choices is also known to contribute to risk for certain psychiatric disorders (eg, addiction/alcoholism) and is characteristic of other psychiatric disorders (eg, attention-deficit hyperactivity disorder) and bipolar disorder (Jentsch and Taylor, 1999; Moeller et al, 2001; Swann, 2010; de Wit, 2009). Therefore, exposure to chronic stress in adolescence may influence the development of the PFC, thus altering cognitive control processes, leading to increased impulsivity and risk for developing psychiatric disorders (Andersen and Teicher, 2009; Chambers et al, 2003). Although the effects of chronic stress in adult animals have been studied extensively, there have been relatively few studies examining the effects of chronic stress in adolescent animals, particularly on measures of impulsive behavior.

Several laboratories have studied chronic stress in adolescence using a variety of stressors including chronic restraint, social isolation, social defeat, and unpredictable stress. Different types of stress can have very selective consequences on behavioral outcomes, but the types of stressors adolescents encounter can include physical, social, and emotional stress. Therefore, it is convenient to use a 
paradigm that mimics aspects of chronic stress stemming from multiple sources. One such model is the chronic corticosterone (CORT) exposure procedure where animals are given CORT via their drinking water, providing a noninvasive means of keeping the primary output hormone of the HPA axis at relatively high circulating levels for many days with predictable, circadian fluctuations (Gourley and Taylor, 2009). Adult animals treated with chronic CORT display a variety of altered behaviors that look very much like chronic exposure to other stressors. These include an increase in depression-like and anhedonic-like behaviors including increased immobility in the forced swim test and a decrease in sucrose preference that can be reversed by antidepressant treatment (Gourley and Taylor, 2009; Gourley et al, 2008a, b). These animals also show deficits in extinction of contextual fear, indicating that they may also have a PTSD-like phenotype (Gourley et al, 2009). Some effects of juvenile/adolescent stress can also be mimicked by CORT exposure alone (Jacobson-Pick and Richter-Levin, 2010). Interestingly, some of the effects of chronic CORT or stress exposure in adult animals do not appear to occur when the stress takes place during adolescence (Bourke and Neigh, 2011; Jankord et al, 2011; Xu et al, 2011). For example, male rodents exposed to chronic restraint or unpredictable stress in adolescence do not show reductions in hippocampal neurogenesis, as is observed in adult animals exposed to chronic stress (Barha et al, 2011; Toth et al, 2008). However, female rodents exposed to chronic stress in either adolescence or adulthood show reductions in neurogenesis (Barha et al, 2011). In addition, chronic CORT exposure in both young (adolescent) and older adult monkeys decreases inhibitory control in a PFC-dependent test of behavioral flexibility (Lyons et al, 2000). However, these studies were done in female monkeys who might be more sensitive to chronic CORT in adolescence. In addition, the monkeys were treated with CORT throughout testing, and the greatest deficits were seen after a prolonged CORT exposure. Few other studies have examined the effects of adolescent stress on cognitive function or impulsivity. Therefore, the present studies used the chronic CORT model in male adolescent rats, and tested impulsivity and other aspects of cognitive function on three well-established tasks, the 5-choice serial reaction time task (5CSRTT), stopsignal reaction time task (SSRTT), and the delay-discounting task (c.f., Evenden, 1999; Winstanley, 2011). In addition, we assessed the sensitivity of the animals to yohimbineinduced increases in impulsivity as this $\alpha 2 \mathrm{a}$-noradrenergic antagonist has been shown to activate the HPA axis and increase impulsivity (Sun et al, 2010; Swann et al, 2005). Yohimbine primarily antagonizes pre-synaptic 2a receptors, resulting in increased release of norepinephrine, and we hypothesized that noradrenergic activity could be modulated by long-term plasticity induced by chronic exposure to CORT in adolescence. Finally, we also examined whether CORT treatment altered the phosphorylation state of two downstream targets of protein kinase A (PKA): the AMPA receptor GluR1 and the transcription factor CREB in the lateral orbital frontal cortex (lOFC), as a previous study indicated that increased CREB phosphorylation in this region is necessary for yohimbine-induced increases in impulsivity (Sun et al, 2010) and this may be mediated through activation of PKA.

\section{MATERIALS AND METHODS}

\section{Subjects}

Male Sprague-Dawley rats were delivered to our animal facility aged 24-26 days (Charles River, Kingston, NY). The rats were allowed approximately 5 days to acclimate to the facility before CORT treatment was initiated at approximately post-natal day (PND) 30. The rats were housed in pairs for the duration of the experiment on a 12:12-h lightdark cycle in a temperature- and humidity-controlled environment. Rats had ad libitum access to food and water except during periods of food restriction described below. All procedures conformed to the policies set forth by the Yale University Institutional Animal Care and Use Committee and the National Institutes of Health Guidelines on the Care and Use of Laboratory Animals.

\section{Chronic Corticosterone Exposure}

Beginning at approximately PND 30, rats were divided into two groups. The first group was treated with 4-pregnen11ß,21-diol-3,20-dione21-hemisuccinate, also known as corticosterone hemisuccinate (CORT) (Steraloids, Newport, RI) for 20 days (until approximately PND50), encompassing the majority of rodent adolescence. The rats received a concentration of $50 \mu \mathrm{g} / \mathrm{ml}$ CORT for the first 14 days of treatment, then the CORT was gradually weaned away by progressively decreasing the concentration to $25 \mu \mathrm{g} / \mathrm{ml}$ for 3 days, then $12.5 \mu \mathrm{g} / \mathrm{ml}$ for 3 more days, and finally switching the rats back to normal tap water for the remainder of the experiments (see Figure 1a for experimental timeline). The second group served as a control and continued to receive normal tap water throughout adolescence and adulthood. These animals were weighed and had their bottles weighed and water changed in the same manner as the CORT-treated group. These methods were almost identical to those described previously (Gourley and Taylor, 2009). The adolescent CORT exposure did not cause any significant differences in weight gain or fluid consumption across adolescence or into adulthood (Figures $1 \mathrm{~b}$ and $\mathrm{c}$ ).

\section{Behavioral Testing}

All rats remained CORT-free during behavioral testing. For all experiments, rats began food restriction 7-10 days after the CORT exposure period had ended at approximately PND 60, which corresponds to early adulthood and allowed time for the HPA axis to recover production of endogenous corticosterone. During food restriction, rats were maintained at $85-90 \%$ of their free-feeding weight. The rats were then trained to respond for $45 \mathrm{mg}$ sucrose pellets (Bio-Serv, Frenchtown, NJ) on one of the three behavioral tasks described below. Separate cohorts of animals were used for testing on each task so that there is no confound of prior behavioral testing. All testing was conducted in standard operant chambers (MedAssociates, St. Albans, VT) and behavioral programs were controlled by MedPC software. All boxes were housed in a sound-attenuating chamber and consisted of Plexiglas front and back walls and ceiling and aluminum sidewalls. The 5CSRTT boxes were extra tall and had one rounded sidewall that contained five apertures 

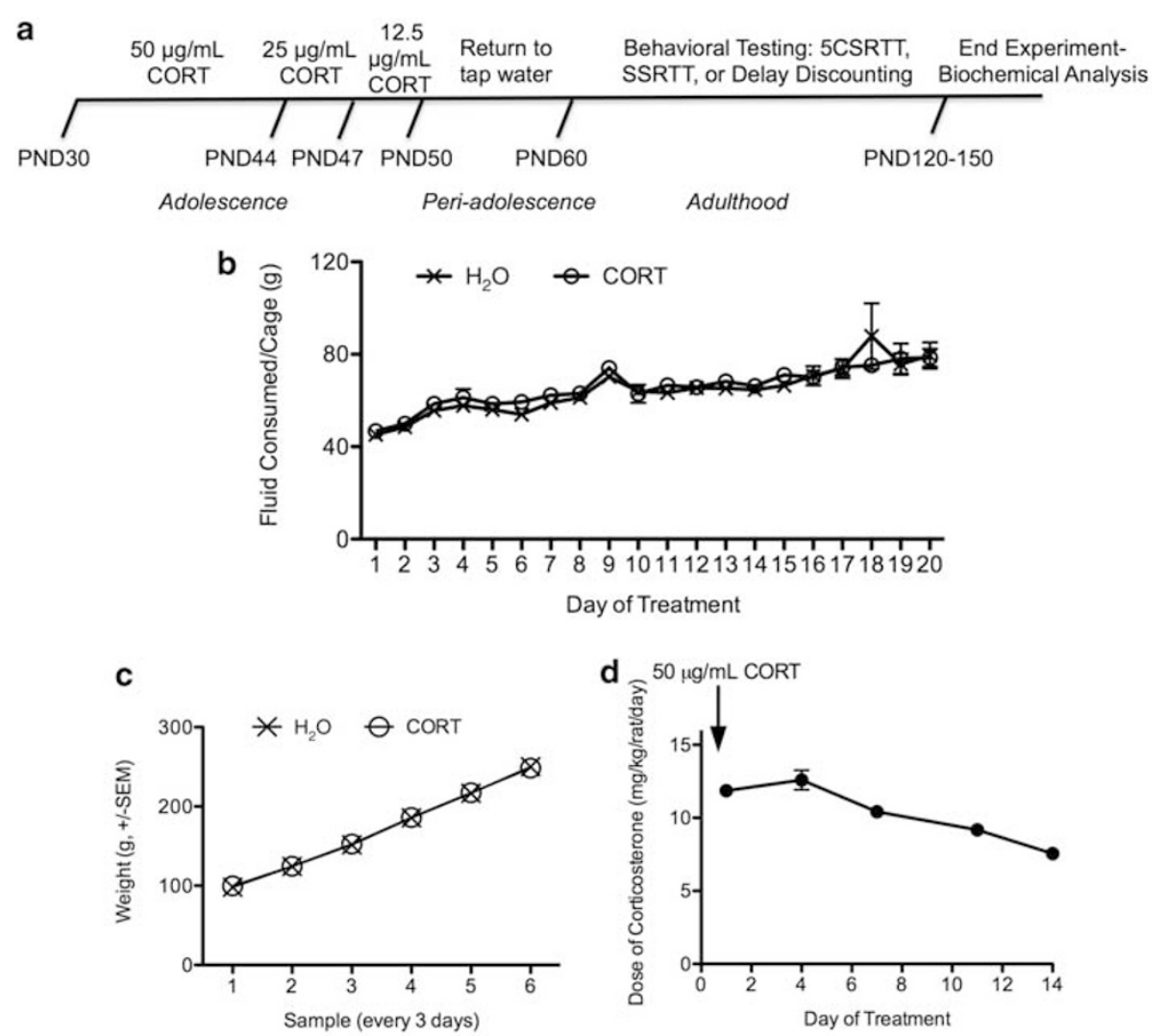

Figure I Timeline of experimental events. (a) Male Sprague-Dawley rats were treated with corticosterone via their drinking water during post-natal days (PNDs) 30-50, and all behavioral testing began at PND 60, 10 days after the CORT exposure. There was no corticosterone CORT given during behavioral testing. (b) The CORT-exposed rats drank the same amount of fluid as rats drinking normal tap water throughout the CORT exposure period. (c) CORTexposed rats did not differ from control rats in the amount of weight gained during the CORT exposure across adolescence. Data are displayed as samples throughout the exposure period. (d) The figure depicts the average dose in $\mathrm{mg} / \mathrm{kg}$ of CORT that rats consumed on selected days throughout the $50-\mu \mathrm{g} / \mathrm{ml}$ phase of exposure.

equipped with lights and sensors to detect when a rat poked his nose into the aperture to break an infrared light beam. The other sidewall consisted of a central port or magazine where sucrose pellets were delivered. The magazine also contained a light and infrared sensors to monitor magazine entries. The boxes used for the SSRTT and delay-discounting task had a flat sidewall with two, rather than five, nose poke apertures that were not used for the experiments, and the other sidewall contained a magazine as described above, and two retractable levers (one placed on either side of the magazine). All boxes were also equipped with a houselight, tone generator, food dispenser, and fan for ventilation and to produce background noise.

5CSRTT. Rats were trained on the 5CSRTT in a similar manner as previously described methods (Chudasama et al, 2003; Jentsch and Taylor, 2003). In brief, rats were trained to make a nosepoke response in a lit nosepoke aperture while ignoring the four unlit apertures. On each trial, one of the five apertures was selected at random by the computer program to be the correct lit aperture.

Initial training. Sessions began with lighting of the magazine. After the rat responded in the magazine, there was a fixed $1 \mathrm{~s}$ intertrial interval (ITI) before the lighting of one aperture. During initial training, the aperture remained lit until the rat responded in one of the apertures. A correct response on the lit aperture resulted in delivery of one sucrose pellet and illumination of the magazine. The rat then had to retrieve the pellet and respond in the magazine again to initiate another trial. An incorrect response in an unlit aperture resulted in a 2-s timeout where the houselight and all other lights were turned off. The timeout ended with illumination of the magazine so that the rat could initiate a new trial. Rats were trained in one $40 \mathrm{~min}$ session per day, with no limit on the number of trials that could be completed. Rats were trained until they made the correct response on at least $70 \%$ of trials.

Remedial training. A few rats from both the adolescent CORT and control groups required additional training to learn the nosepoke response. These rats were given trials where instead of one nosepoke aperture being lit, all five apertures were lit and a response on any of the five resulted in delivery of a sucrose pellet, turning off of the nosepoke aperture lights and turning on the magazine light. Once these rats were making $>100$ reinforced nosepoke responses, they were switched back to the initial training program where only one aperture was lit on a given trial. Rats then had to achieve at least $70 \%$ accuracy on this phase of training before being moved on to the next phase. 
Stimulus duration training. From this point on rats were trained on the same basic program with stimulus durations that were progressively decreased. These sessions lasted for 40 min or until the rat completed 200 trials, whichever came first. These sessions were similar to the initial training described above, except that there was a fixed 2-s ITI between responding in the magazine and lighting of one of the apertures. The aperture was lit for a fixed time (stimulus duration) beginning at $60 \mathrm{~s}$ and progressively decreased to $20 \mathrm{~s}, 5 \mathrm{~s}, 2 \mathrm{~s}$, and $1 \mathrm{~s}$. Rats could make a correct response by nosepoking in the lit aperture while it was illuminated, or during a 5-s limited hold period immediately following the light being turned off. Again, a correct response resulted in the delivery of one sucrose pellet and lighting of the magazine. A response on an unlit aperture was counted as an incorrect response. Failure to respond on any aperture on a trial was counted as an omission. A response made during the 2-s ITI before lighting of an aperture was counted as a premature or impulsive response. Finally, incorrect responses on an unlit aperture that was the previously correct, reinforced aperture on the immediately preceding trial were counted as perseverative responses. Accuracy was calculated as a ratio of correct responses over the total number of responses (ie, omissions were not included in the calculation of accuracy).

Rats had to achieve at least $70 \%$ accuracy at each of the stimulus durations before being moved on to the next shorter stimulus duration. For the longer stimulus durations, this generally only required 1-2 sessions, but at the 1 -sec stimulus duration rats were trained for 5 days regardless of the accuracy achieved.

Variable stimulus duration testing: Rats were then tested for attention and vigilance by varying the length of the stimulus duration within a single session. In these sessions, the stimulus duration was chosen at random for each trial and could be $1.6 \mathrm{~s}, 1.2 \mathrm{~s}$, or $0.8 \mathrm{~s}$. Rats were tested on three daily 40 -min sessions with variable stimulus durations.

Long ITI testing: Next, in order to test the propensity of the rats to make premature, impulsive responses, the stimulus duration was kept constant at $2 \mathrm{~s}$ but the ITI was varied across sessions. Rats were first returned to their prior training of a 2-s stimulus duration and 2-s ITI for one session to insure that the rats remained highly accurate at these parameters. Rats were then switched to a session where the ITI was fixed at $4 \mathrm{~s}$. The rats had three sessions at a 4-s ITI and were then switched to a 6-s ITI for an additional three sessions.

Yohimbine testing: Previous studies have found that yohimbine increases impulsive responses on the 5CSRTT in rats and humans (Sun et al, 2010; Swann et al, 2005). Yohimbine is also anxiogenic and activates the HPA axis (Charney et al, 1984; Myers et al, 2005). Therefore, because the adolescent CORT-treated rats may have differential reactions to a pharmacological stressor, we tested the propensity of yohimbine to increase impulsive responses on the 5CSRTT in these animals. Rats were maintained on a schedule where the ITI was $6 \mathrm{~s}$, but the stimulus duration was reduced to $1 \mathrm{~s}$. Once the rats achieved stable behavior on this schedule, they were given injections of saline and two doses of yohimbine on different days in random order, $10 \mathrm{~min}$ prior to the behavioral testing session. Yohimbine hydrochloride (Sigma-Aldrich, St. Louis, MO) was dissolved in saline at concentrations of $0.625,1.25,2.5$, and $5 \mathrm{mg} / \mathrm{ml}$. Rats were given intraperitoneal injections of two of these doses on different days in a volume of $1 \mathrm{ml} / \mathrm{kg}$ body weight. All rats received a saline injection prior to one day of testing. The doses used have been described previously as being in the behaviorally active range (Sun et al, 2010).

SSRTT. A separate cohort of adolescent CORT-treated and control rats were tested on the SSRTT to assess whether adolescent CORT treatment affected impulsive action, or the ability to inhibit a prepotent response. Rats were trained and tested on this task in a manner very similar to previously published methods (Eagle and Robbins, 2003a, b). Briefly, rats were trained to press two levers in order, in quick succession. On these 'go' trials, a correct response on the second lever resulted in receipt of a sucrose pellet reinforcer. On a subset of trials (stop trials) a stopsignal tone was introduced, indicating that the rat had to withhold responding on the second lever in order to receive reinforcement. The time from the second lever becoming available to presentation of the stop signal varied over testing sessions to obtain a measure of how accurately the rats could withhold responding on stop trials as the signal was presented closer to each individual rat's average time to make a go response (mean reaction time (MRT)) on the task.

One-lever training: In order to initiate lever responding in the task, rats were first trained to press a single lever to receive reinforcement. Each session began with illumination of the houselight, delivery of one sucrose pellet, and lighting of the magazine. The rat then had to initiate the first and each subsequent trial by responding in the magazine. Once the rat made a magazine entry the magazine light would go off, and either the left or right lever (counterbalanced across groups) was inserted into the box. A single response on this lever resulted in reinforcement. Each session terminated after $30 \mathrm{~min}$. Once the rats acquired at least 100 reinforcers in a session, they were moved on to the next phase of training.

Two-lever go training: Go training sessions began in the same way as one-lever training, but a response on the first lever resulted in immediate retraction of that lever and insertion of the opposite lever, rather than reinforcement. Once the second lever was inserted, the rats were initially given $10 \mathrm{~s}$ to make a response. A single lever press resulted in delivery of a sucrose pellet and illumination of the magazine. Failure to make a response resulted in a 5-sec timeout, where the lever was retracted and the houselight turned off. A new trial began with lighting of the magazine and the houselight. Rats initiated a new trial by making a response in the magazine. The sessions lasted for $30 \mathrm{~min}$ or the completion of 200 trials whichever came first. For every session, the average time required for each rat to respond on the second lever after insertion of the second lever was calculated. This value is the MRT. Once rats were reliably responding on the second lever for reinforcement by 
completing $>100$ trials (usually requiring $1-2$ sessions), the time that the second lever was available was reduced to a value $200 \mathrm{~ms}$ greater than each rat's MRT. This time was adjusted for each daily session based on the behavior of each rat on the prior day's session. This training was continued until the rats' MRT became fast (0.75-1.8 s) and stable across sessions with completion of at least $70 \%$ of trials.

Stop signal training: Once the rats were responding accurately and rapidly on go trials, the stop signal trials were introduced. Stop signal training began with the stop signal presented with zero delay. In other words, at the same time that the second lever was presented, a 2-s tone was initiated that signaled to the animal that he needed to withhold responding on the second lever in order to obtain reinforcement. If the rat responded on the second lever on a stop trial then he was given a 5-s timeout as described above. If the rat successfully withheld a response, then the lever was retracted, a sucrose reinforcer was delivered, and the magazine was lit. Stop trials occurred at random on $20 \%$ of trials during a session to maintain accurate go trial responding. There was no difference in the structure of go trials once the stop signal trials were introduced. Rats generally required several days of training to learn to withhold responding on stop trials. Once rats learned to respond with at least $65 \%$ accuracy on both stop and go trials, they were moved to the stop signal-testing phase. Only a subset of rats learned to accurately respond on both stop and go trials, so three separate cohorts of adolescent CORT-treated and control rats were tested on the SSRTT in order to obtain sufficient statistical power for analysis.

Stop-signal testing: During stop-signal testing, the time delay between the second lever being presented and the initiation of the stop signal tone was varied across sessions to occur either $800,600,400,200$, or $100 \mathrm{~ms}$ prior to each rat's own MRT from the previous day. In other words, the stop-signal delay (SSD) for $800 \mathrm{~ms}$ was calculated for each individual rat according to the following equation:

$$
\mathrm{SSD}=\mathrm{MRT}-0.800 \mathrm{~s}
$$

Each rat began stop signal testing with a delay set to $800 \mathrm{~ms}$ before his MRT to ensure that the rat could maintain accurate stop trial responding when the initiation time of the stop signal was varied. After testing at $800 \mathrm{~ms}$, the SSD was varied across daily sessions in random order from 100$600 \mathrm{~ms}$ prior to each rats' MRT. Once rats completed sessions at every SSD, they were re-tested at zero delay to verify that the animals could still accurately respond on both stop and go trials. Any rat that could not maintain $>65 \%$ accuracy on go trials at every SSD delay tested was not included in the analysis. Removing these rats from the analysis was necessary because a fraction of rats would change their response strategy at varying delays and elect to stop responding on go trials in order to achieve higher accuracy on stop trials. Inclusion of these animals could have skewed these data toward inappropriately high stop trial accuracy.

Estimation of stop-signal reaction time: We estimated the stop-signal reaction time (ie, the amount of time it takes for the stopping process to finish after the onset of the stop signal) using the method described by Eagle and Robbins (2003b). The estimated stop-signal reaction time was calculated for each rat at the $800,600,400$, and $200 \mathrm{~ms}$ delays, as these corresponded to the linear portion of the stop function that passed through 50\% accuracy. The average of these four values was then calculated for each rat and taken as the strongest estimate of their stop-signal reaction time. These values were then averaged for each group.

\section{Delay Discounting}

Finally, another cohort of adolescent CORT-treated and control rats was tested on the delay-discounting task as a measure of impulsive choice. The behavioral paradigm was based on the previously published protocols (Mar and Robbins, 2007; Mar et al, 2011). Briefly, rats were trained to press one lever for delivery of one sucrose pellet and to press the opposite lever for delivery of four sucrose pellets. The delivery of one pellet always occurred immediately after making the lever press, but pressing the lever for four pellets resulted in delivery of the pellets after varying delays. In this manner, the task assesses each animal's willingness to wait for a large reinforcer, rather than choosing a smaller, immediate reinforcer. Rats were first trained to lever press for reinforcement in general and to become accustomed to long ITIs before introducing delays.

Lever press training: In the initial phase of training, each session began with lighting of the houselight and the magazine light. The rat had to respond in the magazine to initiate a trial. Once the rat made a magazine response, the magazine light was extinguished and either the left or right lever was inserted into the chamber at random. A single lever press resulted in delivery of one sucrose pellet, retraction of the lever, and illumination of the magazine. Failure to make a lever response within $30 \mathrm{~s}$ resulted in retraction of the lever and a 5-s timeout where the houselight was extinguished. On the next trial, the opposite lever was inserted into the magazine and a single response resulted in delivery of one sucrose pellet. The first lever to be inserted on each pair of trials was randomly chosen, but the rats had an equal number of trials where the left or right lever was available. Sessions lasted for $30 \mathrm{~min}$ or until 100 trials were completed. Once rats learned to respond on the majority of trials for both the left and right lever, they were moved on to the next phase of training.

Training on increased ITI: The next phase of training was essentially the same as initial lever press training except that a 40-s ITI was introduced at the end of each trial, regardless of whether the rat made a lever response or not. If the rat made a response on the inserted lever then he received a single sucrose pellet, the magazine was lit, and the lever was retracted. After responding in the magazine to consume the pellet, the magazine light and houselight were extinguished for $40 \mathrm{~s}$. At the end of the ITI, the magazine light was turned on and a response initiated a new trial, which began with lighting of the houselight, insertion of one of the two levers, and extinction of the magazine light. If the 
rat failed to respond on the lever within $10 \mathrm{~s}$, then the houselight was extinguished and the lever was retracted. After $40 \mathrm{~s}$, the magazine light was lit to signal that a new trial could be initiated. The sessions lasted for $60 \mathrm{~min}$ or until 90 trials were completed. Once rats were successfully responding on the majority of trials, they were moved on to the next phase of training.

Lever discrimination and delay-discounting training: The next phase of training involved training the rats that one lever (always the left or the right, counterbalanced across groups) was associated with immediate delivery of one pellet, whereas the other lever was associated with delivery of four pellets at varying delays. These sessions consisted of five blocks of 12 trials each. Each block of 12 trials represented different delays to delivery of the four pellets if the large reinforcer lever was chosen. Each successive block of trials used a progressively increasing delay for delivery of the four pellets in the following order: $0 \mathrm{~s}, 10 \mathrm{~s}, 20 \mathrm{~s}, 40 \mathrm{~s}$, and $60 \mathrm{~s}$. The rat had to make a response in the magazine to begin each trial as before. Each block of trials began with two forced-choice trials where either the left or right lever only was inserted on the first of these two trials, chosen at random, and the opposite lever was inserted on the next forced-choice trial. A response on the inserted lever resulted in reinforcement under the conditions for that block of trials for that lever. In other words, if the left lever was paired with immediate delivery of one pellet, then pressing the left resulted in this outcome. On the next forced-choice trial, only the right lever would be available and a response on this lever would result in four pellets delivered after the delay that was operating during that block of trials. The next 10 trials in the block were freechoice trials where on each trial both levers were inserted into the chamber and the rat could choose to respond on either lever, with the left always giving immediate reinforcement of one pellet and the right always giving four pellets after the set delay. For all trials, rats had $10 \mathrm{~s}$ to initiate a trial after the magazine was lit, otherwise the light was extinguished and a new ITI began. The number of missed trials was recorded as an estimate of attention and motivation to perform the task. Likewise, rats were given $10 \mathrm{~s}$ to respond on a lever on each trial, or the lever was retracted, the houselight was extinguished, and a new ITI began. Lever omissions were also recorded. During this phase of the experiment, the ITI was set at $90 \mathrm{~s}$. The session continued until all 60 potential trials were completed (roughly $110 \mathrm{~min}$ ). Successful training in the task was indicated by rats efficiently discriminating between the levers associated with small and large reinforcement with $0 \mathrm{~s}$ delay. Rats that understand the task should preferentially choose the large reinforcer when there is no delay to reinforcer delivery. Rats demonstrating a side bias (ie, always choosing the left lever regardless of reward magnitude or delay) were switched to the opposite leverreinforcer size contingency, and if the rats still did not adjust their behavior then they were excluded from the analysis.

Short delay testing: After the rats reached stable behavior for several days at these delays (16 total sessions), they were switched to a series of shorter delays as follows: $0 \mathrm{~s}, 3 \mathrm{~s}, 6 \mathrm{~s}$,
$10 \mathrm{~s}$, and $15 \mathrm{~s}$, in order to assess whether rats would choose the larger reinforcer more frequently if there was a shorter delay. All other aspects of the task remained exactly as described above. Rats were maintained on this version of the task for 10 sessions.

\section{Biochemical Studies}

The methods used for biochemical analysis were similar to those previously published in our laboratory (Gourley et al, 2009). At the end of behavioral testing on the 5CSRTT, rats were killed by rapid decapitation and the brains were rapidly dissected and frozen on dry ice. Brains were stored at $-80{ }^{\circ} \mathrm{C}$ until dissection by cutting frozen brains into $2 \mathrm{~mm}$ slices using a metal brain mold (Plastics One, Roanoke, VA). Specific brain regions were then obtained from the slices using a tissue punch $(1.2 \mathrm{~mm}$ diameter, Fine Science Tools, Foster City, CA) including the lateral region of the lOFC. The tissue punches were stored at $-80^{\circ} \mathrm{C}$ until the tissue could be homogenized using a sonicator in a buffer containing $1.37 \mathrm{mM} \mathrm{NaCl}, 20 \mathrm{mM}$ TrisHCl, 1\% Igepal, 10\% glycerol, and 1:100 phosphatase inhibitor cocktails I and II (Sigma-Aldrich). Protein concentrations in each sample were then quantified using the Pierce bicinchoninic acid assay kit (ThermoScientific, Rockford, IL). For immunoblotting, $10 \mu \mathrm{g}$ of protein from each sample was diluted $4: 1$ in 1\% SDS and loading buffer containing $20 \%$ glycerol, 2\% SDS, and bromophenol blue, and the samples were then boiled for $10 \mathrm{~min}$. Samples were loaded onto 1020\% Tris-glycine gradient gels (Invitrogen, Carlsbad, CA) and proteins were separated using electrophoresis. Proteins were then transferred to nitrocellulose membranes and incubated in the following primary antibodies: antiphoshpho Ser133 CREB (Rb, 1:1000, Sigma-Aldrich, St. Louis, MO), anti-CREB (Rb, $1: 1000$, Sigma), anti-phospho Ser845 GluR1 (Rb, $1: 1000$, Phosphosolutions, Aurora, CO), anti-GluR1 (Rb, 1:500, Millipore, Billerica, MA), and antiGAPDH (Ms, $1: 10,000$, Advanced Immunochemicals, Long Beach, CA). Immunoblots were incubated in the following secondary antibodies for analysis on the LI-COR Odyssey Imager: IRDye700 Dx Anti-Rb IgG and IRDye800 Dx AntiMs IgG (1:5000, Rockland Immunochemicals, Glibertsville, PA). Protein bands were identified and analyzed using Licor-Odyssey software. The expression of each protein was normalized to GAPDH expression for that sample, and for each gel, bands were further normalized to the average of the $\mathrm{H}_{2} \mathrm{O}$-treated group to control for between gel variations.

\section{Statistical Analysis}

For the majority of the statistical analysis, a repeatedmeasures ( $\mathrm{rm}$ ) ANOVA was used where the betweensubjects factor was adolescent CORT or $\mathrm{H}_{2} \mathrm{O}$ treatment whereas the within-subjects factor was stimulus duration, ITI, yohimbine dose, SSD, or delay to larger reinforcer, as appropriate for each test. Significance was set at $\alpha$ of 0.05 , and any significant interaction effects were further analyzed by Bonferroni's post hoc test. Western blot data were quantified and analyzed by an unpaired, $t$-test with $\alpha$ set to 0.05 . 


\section{RESULTS}

\section{Corticosterone Exposure}

Data from all experiments was combined to analyze whether CORT exposure affected fluid consumption or weight gain across adolescence. These data were combined because there were no significant differences between the different cohorts of animals. During the entire CORT exposure period, each cage of rats consumed the same amount of fluid whether they were drinking CORT-containing water or regular tap water, as indicated by no significant effect of solution or interaction when analyzed by rmANOVA $(p=0.24 ; p=0.95$, respectively). There was a significant effect of day indicating that as the animals grew, their total fluid consumption also increased $\left(\mathrm{F}_{(1,19)}=13.52, p<0.001\right.$; Figure 1b). Likewise, weight was sampled across the CORT exposure period and Figure 1c indicates that CORT had no significant effect on adolescent weight gain $(p=0.79)$, but that weight significantly increased over time $(p<0.001)$. Figure $1 \mathrm{~d}$ illustrates the total dose of CORT in $\mathrm{mg} / \mathrm{kg}$ per rat per day consumed at days sampled during the $50 \mu \mathrm{g} / \mathrm{ml}$ phase of CORT exposure, indicating that the level of CORT the rats were exposed to ranged from approximately 12.5$7.5 \mathrm{mg} / \mathrm{kg}$ across days and decreased in direct relation to their increase in weight. The total dose at the end of the exposure period is similar to that previously reported for adult rats, and that maintains high levels of circulating CORT (Gourley and Taylor, 2009; Gourley et al, 2008a).

\section{CSRTT - Stimulus Duration Testing}

Eight adolescent CORT-treated and eight $\mathrm{H}_{2} \mathrm{O}$-treated control rats were trained on the 5CSRTT beginning 10 days after the cessation of CORT treatment and PND60 (Figure 1a). Rats acquired accurate responding on the task at roughly the same rate, with $7 / 8 \mathrm{CORT}$ rats and $6 / 8 \mathrm{H}_{2} \mathrm{O}$ rats responding with $>70 \%$ accuracy at the 5-s stimulus duration on the 10th total day of training (all phases). The $\mathrm{H}_{2} \mathrm{O}$ control rats required 3 and 5 days, respectively, of additional training to reach the same criteria, and the CORT rat required four additional days of training. From that point on all rats progressed at about the same rate, indicating that prior adolescent CORT treatment did not produce a difference in ability to learn the task.

Figure 2 illustrates the results of varying the stimulus duration on accuracy, omissions, premature, and perseverative responses when rats were tested at stable stimulus durations of 5, 2, or $1 \mathrm{~s}$ each session, and accuracy when rats were tested at variable stimulus durations (1.6, 1.2, and $0.8 \mathrm{~s})$. A rmANOVA of accuracy across stimulus durations revealed a main effect of stimulus duration $\left(\mathrm{F}_{(2,28)}=57.31\right.$, $p<0.001)$, but no significant effect of treatment $(p=0.51)$ or interaction $(p=0.89)$, indicating that although all rats showed reduced accuracy as the stimulus duration decreased and attentional demands increased, there was no effect of adolescent CORT treatment (Figure 2a). Likewise, when rats were tested at variable stimulus durations within a session, analysis of accuracy by rmANOVA revealed a main effect of stimulus duration $\left(\mathrm{F}_{(2,28)}=7.63, p=0.002\right)$, but no effect of treatment $(p=0.48)$ or interaction $(p=0.81)$. Again, these data indicated that although decreasing the stimulus duration does decrease accuracy as expected, there is no effect of adolescent CORT treatment on performance in the task (Figure $2 \mathrm{~b}$ ).

Analysis of premature responses again indicated a main effect of stimulus duration $\left(\mathrm{F}_{(2,28)}=9.96, p=0.005\right)$, and although there was no significant effect of treatment $\left(\mathrm{F}_{(1,28)}=2.79, \quad p=0.12\right), \quad$ or interaction $\quad\left(\mathrm{F}_{(2,28)}=2.65\right.$, $p=0.09)$; there was a strong trend toward adolescent CORT treatment reducing premature responses, particularly at the 1-s stimulus duration (Figure 2c). Analysis of omissions and perseverative responses revealed a main effect of stimulus duration $\left(\mathrm{F}_{(2,28)}=7.18, p=0.003 ; \mathrm{F}_{(2,28)}=18.08, p<0.001\right)$, but no effect of treatment $(p=0.63 ; p=0.43)$ or interaction $(p=0.68 ; p=0.41)$, respectively, for each measure (Figures $2 \mathrm{~d}$ and e). Finally, there were no differences between CORTtreated and control rats on their latency to make a response or initiate new trials (data not shown).
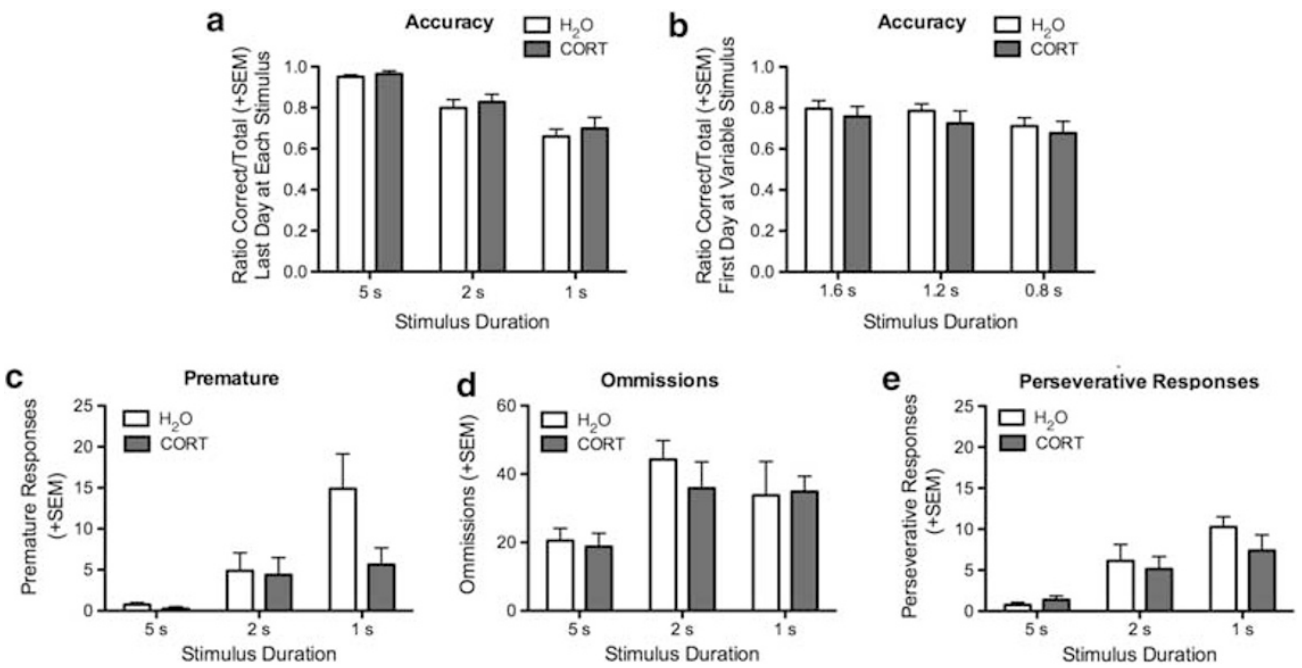

Figure 2 Effects of chronic adolescent corticosterone (CORT) on baseline behaviors in the 5-choice serial reaction time task (5CSRTT). Chronic adolescent CORT had no significant effects on task accuracy at stable stimulus durations (a) or variable stimulus durations (b), though all rats showed decrements in performance as stimulus duration decreased. Chronic adolescent CORT also produced no significant differences in other measures of task performance including premature responses at a stable intertrial interval (ITI) (c), trial omissions (d), or perseverative responses (e). 


\section{CSRTT - ITI Testing}

In order to determine whether there might be a real difference in premature or impulsive responding between the adolescent CORT-treated and control-treated rats, we next tested the animals at increasing ITIs. The rats were first returned to a stable stimulus duration of $2 \mathrm{~s}$ and an ITI of $2 \mathrm{~s}$, for which they had been well trained. Then, on the next daily session, the rats were switched to an ITI of $4 \mathrm{~s}$, which forced the animals to wait longer than they were accustomed to find out which nose-poke port was the correct one to make a response for reinforcement. Increasing the ITI is known to produce an increase in premature responses (Chudasama et al, 2003). The rats were kept at a 4-s ITI for a total of three sessions, and then it was increased again to $6 \mathrm{~s}$ to again force the animals to wait longer than they were accustomed to make an accurate response. Figure 3 illustrates the performance of rats on the re-baseline day at a 2-sec stimulus duration and $2 \mathrm{~s}$ ITI, followed by performance on the first day the animals were switched to a 4-s ITI and the first day they were switched to a 6-s ITI. All rats improved their accuracy and decreased premature responding after multiple days of training on a new ITI, so these data reflect the ability of the rats to adjust to novel changes in task demands. As expected, rmANOVA revealed a main effect of ITI on accuracy in the task $\left(\mathrm{F}_{(2,28)}=261.8, p<0.001\right)$, as the rats made many more premature responses and often missed the stimulus light. There was a strong trend for a main effect of adolescent CORT treatment $\left(\mathrm{F}_{(1,28)}=4.13, p=0.061\right)$ on accuracy in the task, but there was no significant interaction $(p=0.30)$. However, when accuracy at the 2-s ITI was removed from analysis in order to specifically assess differences in accuracy in the face of changing task demands, as opposed to baseline behavior that had been well-trained, we found a significant effect of adolescent CORT treatment $\left(\mathrm{F}_{(1,14)}=5.43, p=0.035\right)$, but no significant interaction, indicating that although at baseline adolescent CORT treatment did not affect accuracy, prior CORT treatment does result in a slight improvement in performance in the face of novel task demands (Figure 3a).

The adolescent CORT treatment also improved performance in the task in terms of reducing impulsive, premature responses. Figure $3 \mathrm{~b}$ illustrates that there was a significant main effect of prior CORT on the number of premature responses $\left(\mathrm{F}_{(1,28)}=7.62, p=0.015\right)$, as well as a main effect of ITI $\left(\mathrm{F}_{(2,28)}=96.84, p<0.001\right)$, but no significant interaction, though there was a strong trend $(p=0.068)$. The effect of adolescent CORT treatment was also significant when the 2-s stimulus duration was excluded from analysis. Therefore, adolescent CORT treatment reduced the tendency of rats to respond prematurely in response to increases in the ITI. Analysis of omissions revealed a significant effect of ITI $\left(\mathrm{F}_{(2,28)}=12.20\right.$, $p=0.002)$, but no significant effect of treatment $(p=0.17)$ or interaction ( $p=0.86$; Figure $3 c)$. Likewise, although there was a significant effect of ITI on perseverative responses $\left(\mathrm{F}_{(2,28)}=5.61, p=0.009\right)$, there was no effect of treatment $(p=0.80)$ or interaction $(p=0.67)$. Although in general, increasing the ITI worsens performance on the task, increasing the ITI actually decreases perseverative responses. This was likely due to the fewer correct responses that the rats made overall, and possibly because of an inability of the rats to maintain in working memory the identity of the previously reinforced aperture over longer delays. Regardless, prior adolescent CORT treatment did not significantly affect perseverative responding at changing ITIs (Figure 3d).

\section{CSRTT - Yohimbine Dose-Response Curve}

The $\alpha$-2-noradrenergic receptor antagonist yohimbine has been shown to increase impulsive responding on the
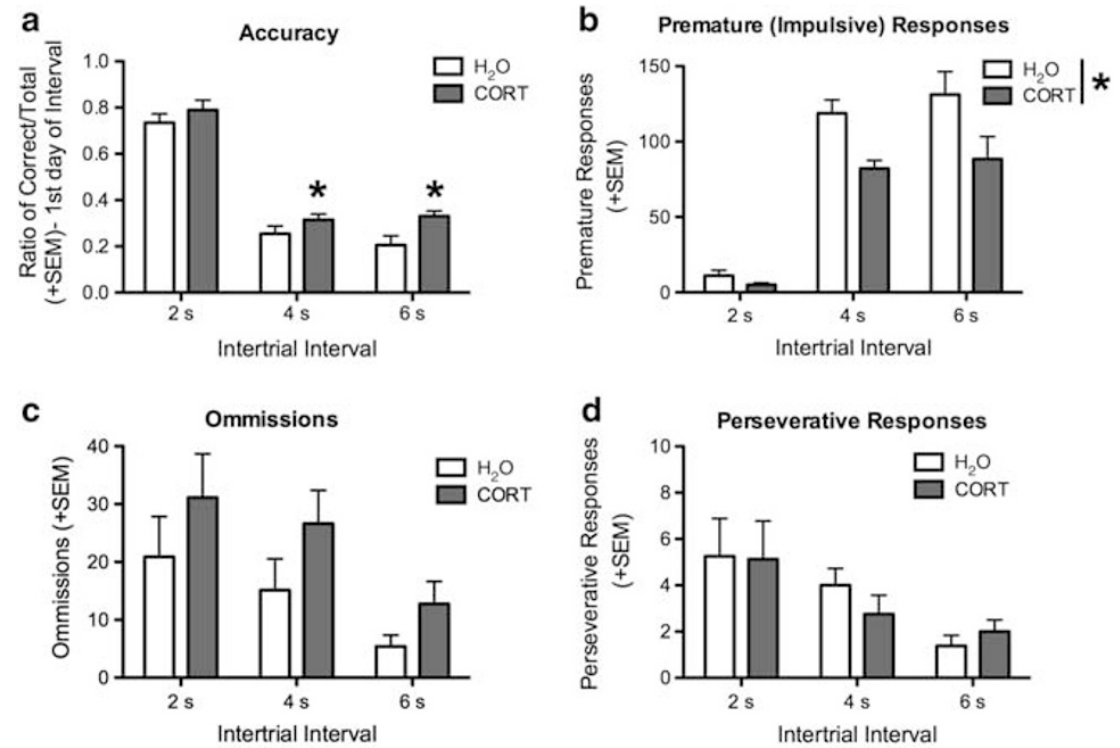

Figure 3 Chronic adolescent corticosterone (CORT) decreases impulsivity with changing intertrial intervals (ITIs) on the 5-choice serial reaction time task (5CSRTT). Chronic adolescent CORT improved task accuracy when the ITI was switched to both 4 and $6 \mathrm{~s}$ (a), and produced an overall reduction in premature responses on the first day that the ITI was switched (b). The adolescent CORT-treated rats showed no significant differences in omissions (c) or perseverative responses (d) as the ITI increased. ${ }^{*} p<0.05$. 

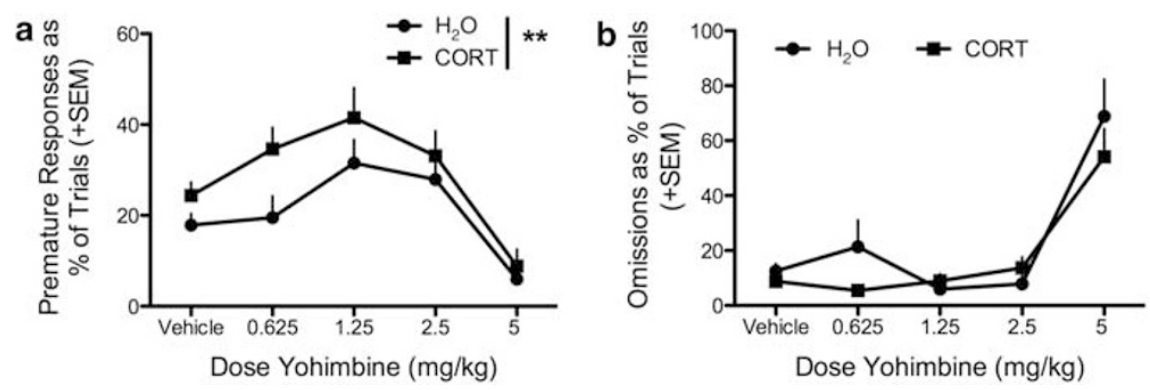

Figure 4 Chronic adolescent corticosterone (CORT) treatment enhances sensitivity to yohimbine-induced impulsivity on the 5-choice serial reaction time task (5CSRTT). Adolescent CORT-treated and control rats were given injections of saline and varying doses of yohimbine prior to testing on the 5CSRTT with a stable intertrial interval (ITI) and stimulus duration. Adolescent CORT-treated rats had an upward and leftward shift in the yohimbine doseresponse curve, indicative of a greater sensitivity to yohimbine's effects on impulsivity (a), whereas the number of omissions on the task was not different between groups and was not significantly disrupted except at the highest dose of yohimbine (b). *** $<0.0$ l.

5CSRTT in rats and humans (Sun et al, 2010; Swann et al, 2005), and because chronic CORT exposure during adolescence could alter brain responsiveness to noradrenergic stimulation and other stressors in adulthood, we examined the effect of yohimbine at several doses on behavior in the 5CSRTT. Figure 4 illustrates the effects of yohimbine on premature responses and omissions as a percentage of trials initiated. Yohimbine, particularly at the highest dose, altered the number of trials the rats would actually initiate, as opposed to data presented above, where all rats initiated close to all possible trials. Analysis of the percent of trials resulting in a premature response by ANOVA revealed a main effect of dose $\left(\mathrm{F}_{(4,86)}=11.67\right.$, $p<0.001)$ and a main effect of adolescent CORT treatment $\left(\mathrm{F}_{(1,86)}=8.18, p=0.005\right)$, but no interaction $(p=0.70)$. The lower doses of yohimbine increased premature responding in all animals, but the CORT-treated animals showed a greater amount of impulsive responding, as indicated by the main effect of CORT (Figure $4 \mathrm{a}$ ). Both groups of animals showed a great reduction in impulsivity at the $5-\mathrm{mg} / \mathrm{kg}$ dose, and this was largely because of an overall disruption in behavior in the task. For example, analysis of omissions revealed a main effect of dose $\left(\mathrm{F}_{(4,86)}=28.67, p<0.001\right)$, but no effect of treatment $(p=0.17)$ or interaction $(p=0.27)$, illustrating that the $5-\mathrm{mg} / \mathrm{kg}$ dose greatly increased omissions in the task in both groups, whereas the other doses of yohimbine had relatively little impact on completion of trials in the task (Figure 4b). Similarly, accuracy was not altered by yohimbine when compared with a vehicle injection except at the $5-\mathrm{mg} / \mathrm{kg}$ dose where accuracy decreased. In addition, perseverative responses increased at the $5-\mathrm{mg} / \mathrm{kg}$ dose, but only in the subset of animals of both treatment groups that made any perseverative responses at all at that dose (data not shown).

\section{CSRTT - Biochemical Analysis}

A study by Sun et al (2010) found that the yohimbineinduced increase in impulsivity on the 5CSRTT was associated with an acute increase in phosphorylation of CREB in the 1OFC, and that inactivation of CREB could prevent yohimbine-induced increase in impulsivity. Therefore, we examined whether baseline differences in phosphoCREB in the adolescent CORT-treated rats could explain the observed differences in impulsivity, either in response to changing ITI's or to yohimbine administration. We also examined the expression of the phosphorylated form of the AMPA receptor subunit GluR1 at serine 845 , as this is also a target of cAMP-dependent PKA. We analyzed protein expression using eight rats per group. Analysis of phosphoand total CREB expression, and the ratio of phospho- to total CREB revealed no significant differences between the adolescent CORT-treated and control animals $(p=0.36$, 0.27 , and 0.49 , respectively; Figure $5 \mathrm{a}$ ). However, analysis of phospho-GluR1 expression revealed a significant increase in the adolescent CORT-treated rats over controls $(p=0.028)$. Although there was no significant increase in the expression of total GluR1, there was a trend $(p=0.087)$, and because there was no significant difference in the ratio of phosphoto total GluR1 expression $(p=0.484)$, it is likely that the increase in phospho-GluR1 represents an overall increase in GluR1 in the 1OFC after adolescent CORT treatment that is maintained in the more active, phosphorylated state (Figure 5b). An increase in active GluR1 could represent a mechanism by which adolescent CORT-treated rats are more sensitive to yohimbine-induced impulsivity.

\section{SSRTT}

The reduction in premature responses at increasing ITIs produced by adolescent CORT exposure on the 5CSRTT could be interpreted as an improved ability to wait for the stimulus light to appear, or as an improved ability to inhibit ongoing responses. In order to tease apart these two possibilities additional rats were tested on the SSRTT, which gives an estimate of the ability to inhibit prepotent responding, and the delay-discounting task, which assesses willingness to wait for larger reinforcement. First, a separate cohort of rats were treated with CORT or maintained on regular $\mathrm{H}_{2} \mathrm{O}$ during adolescence. Beginning 10 days after the end of CORT exposure (PND60), rats were trained to perform the SSRTT. Overall, a lower percentage of rats successfully reached the learning criteria of this task (ie, $>65 \%$ accuracy on go trials and stop trials with zero delay); therefore, a total of $24 \mathrm{H}_{2} \mathrm{O}$ and $24 \mathrm{CORT}$ rats were trained on the task in order to have final group sizes of $15 \mathrm{H}_{2} \mathrm{O}$ and 11 CORT-treated animals, which allowed sufficient power for statistical analysis.

Figure 6 illustrates the behavior of the rats that did successfully learn the task. A rmANOVA of accuracy on stop trials across the varying SSDs indicated a main effect of treatment $\left(\mathrm{F}_{(1,96)}=4.45, p=0.045\right)$, illustrating that the 
a

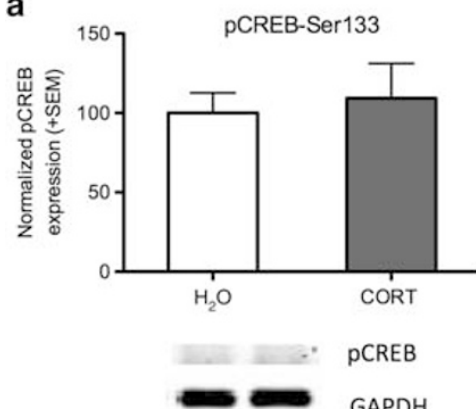

CORT $\mathrm{H}_{2} \mathrm{O}$

b

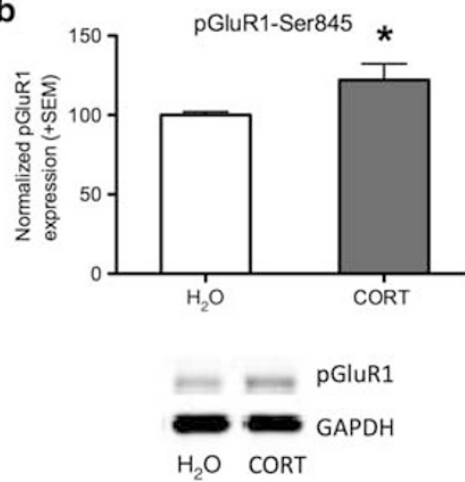

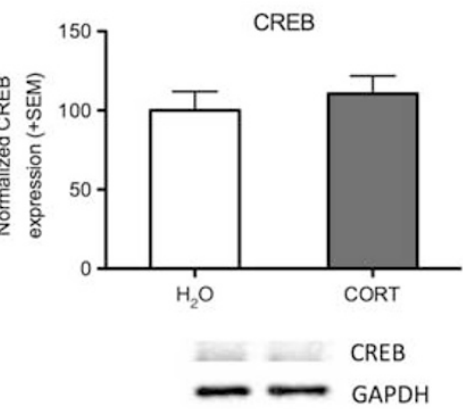

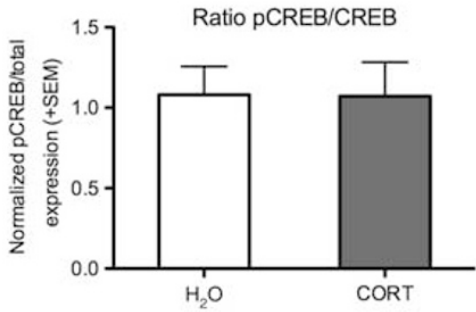

CORT $\mathrm{H}_{2} \mathrm{O}$
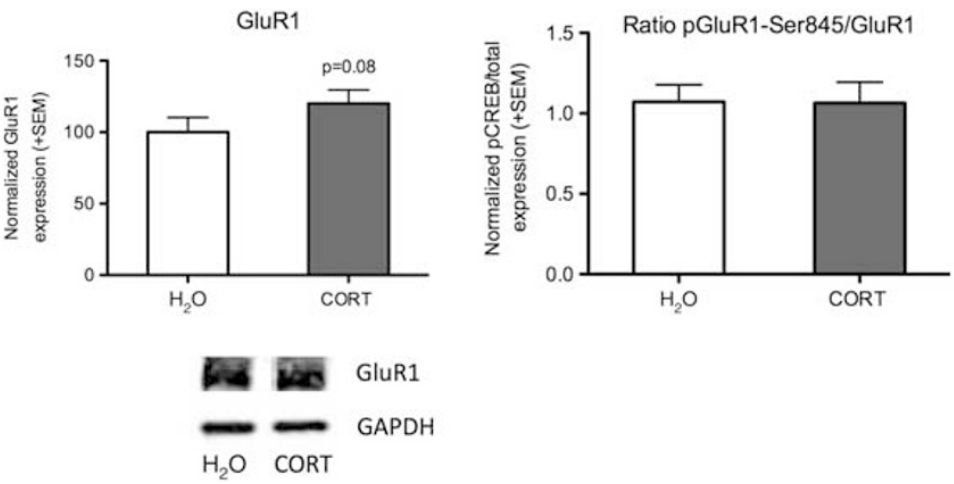

Figure 5 Chronic adolescent corticosterone (CORT) treatment does not alter expression of CREB, but increases phosphorylated GluRI in the lateral orbital frontal cortex (IOFC). Phosphorylation of CREB in the IOFC is associated with yohimbine-induced impulsivity on the 5-choice serial reaction time task (5CSRTT) (Sun et al, 20 I0), but adolescent CORT treatment did not alter the expression of phospho-CREB at ser I33, total CREB, or the ratio of phosphoto total CREB in the IOFC (a). Adolescent CORT treatment did significantly increase phosphorylation of the AMPA receptor subunit GluRI at the protein kinase A (PKA) site ser845, with a trend toward increasing overall GluRI expression, $p=0.08$. However, the ratio of phosphorylated to total GluR I was not different, indicating a likely overall increase in protein expression that is maintained in the phosphorylated state (b). All bands were normalized to GAPDH and representative blots are below the graphical quantification, ${ }^{*} p<0.05$.
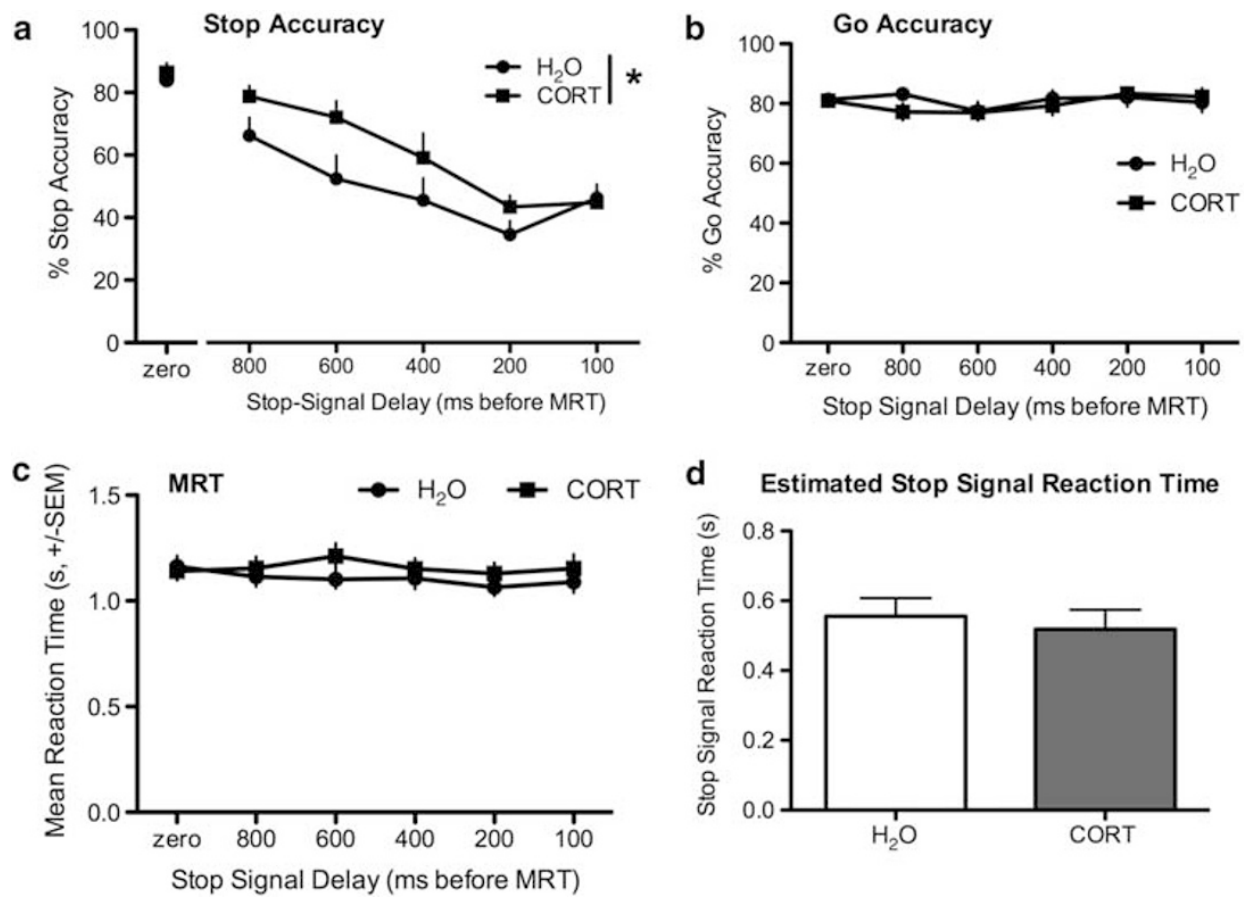

Figure 6 Chronic adolescent corticosterone (CORT) improves accuracy on the SSRTT. Adolescent CORT-treated rats demonstrated better stop accuracy across increasing stop signal delays (SSDs), but were not different from controls at baseline stop accuracy with zero delay (a). Adolescent CORT treatment did not affect accuracy on go trials at any SSD (b). CORT treatment did not affect the mean reaction time (MRT) on go trials at any delay (c). CORT treatment did not significantly alter the estimated stop-signal reaction time (d), ${ }^{*} p<0.05$. 
adolescent CORT-treated rats were actually more accurate at withholding responses on stop trials across the SSDs compared with the $\mathrm{H}_{2} \mathrm{O}$-treated controls. There was also a main effect of SSD as expected, with all animals performing less accurately on stop trials as the delay was increased and got closer to each animal's mean go reaction time $\left(F_{(4,96)}=17.03, p<0.001\right)$, and there was no significant interaction $(p=0.26)$, indicating that the CORT-treated animals were more accurate overall, rather than at any specific SSD (Figure 6a). There was no significant difference between the CORT and $\mathrm{H}_{2} \mathrm{O}$ groups on stop accuracy at zero delay to the stop signal using an unpaired $t$ test $(p=0.68)$, indicating that there was no innate difference in ability to withhold responding on stop trials when task demand was low. The difference produced by adolescent CORT treatment only was manifested as the difficulty of the task increased (Figure 6a). Likewise, analysis of accuracy on go trials across all of the SSDs by rmANOVA revealed no effect of delay $(p=0.14)$, treatment $(p=0.99)$, or interaction $(p=0.40)$, indicating that neither adolescent CORT treatment or varying the SSD altered the ability of rats to accurately respond on go trials in the task (Figure 6b). Analysis of the mean go reaction times across SSDs indicated that there was no significant effect of treatment $(p=0.47)$, suggesting that CORT's effects on stop accuracy were not because of overall slowing of responses (Figure 6c). In addition, analysis of the estimated stopsignal reaction time by a one-tailed $t$-test indicated no significant difference between groups in stop-signal reaction time ( $p=0.32$; Figure $6 \mathrm{~d})$.

\section{Delay-Discounting Task}

Although the results of the SSRTT indicate that adolescent CORT exposure reduced impulsive action, they do not address whether adolescent CORT exposure affects impulsive choice/decision making. Therefore, we tested a novel cohort of rats on the delay-discounting task, a procedure that requires animals to choose between immediate, small reinforcement and delayed, larger reinforcement to assess impulsive decision-making ability. A group of eight adolescent CORT-treated and eight $\mathrm{H}_{2} \mathrm{O}$ treated control rats were initially trained on the task. One CORT-treated rat and one $\mathrm{H}_{2} \mathrm{O}$-treated rat developed a lever bias, such that they responded on the same lever (left or right), regardless of whether it was associated with immediate or delayed reinforcement. These animals were excluded from data analysis, so that the final group sizes were seven adolescent CORT-treated and seven adolescent $\mathrm{H}_{2} \mathrm{O}$-treated. Rats were initially trained at delays to reinforcement ranging from 0-60 s. Both groups required approximately 12 sessions of training to reach stable choice responding at these delays. The rats continued on this schedule for four additional sessions to insure behavioral stability. Figure 7 illustrates the discounting functions for both groups as percent choice of the large, delayed reinforcer across the various delays. The function is based on the average percent choice of the larger reinforcer from the last 3 days of training for each rat at each delay, which is how delay-discounting data has been reported in previous studies (Mar and Robbins, 2007). Figure 7a illustrates responding early in training before rats show a strong preference for the larger reinforcer at zero delay. There was no significant effect of treatment early in training $(p=0.27)$. After stable responding was established, analysis by ANOVA revealed a significant effect of delay $\left(\mathrm{F}_{(4,60)}=\right.$ $106.6, p<0.001)$, indicating that as the delay to reinforcement got longer, all rats switched preference to the small, immediate reinforcer, but there was no effect of treatment $(p=0.14)$ or significant interaction $(p=0.44$, Figure $7 \mathrm{~b})$.

Owing to the very steep discounting functions observed at these delays, with rats only choosing the large reinforcer at the shortest delay of $10 \mathrm{~s} 20-30 \%$ of the time, we decided to test shorter delays ranging from 3-15 s to see whether group differences could be observed when rats did not have to wait so long for the larger reinforcer. A statistical difference in choice behavior between the groups was observed after 5 days of training at the new delays, with a significant main effect of both treatment $\left(\mathrm{F}_{(1,60)}=12.46, p<0.001\right)$ and delay $\left(\mathrm{F}_{(4,60)}=29.40, p<0.001\right)$, with both groups preferring the large reinforcer over $80 \%$ of the time with zero delay, but with the CORT-treated group choosing the larger reinforcer significantly less frequently if there was any delay to reinforcement (Figure $7 \mathrm{c}$ ). The rats were trained for an additional five sessions and the statistical difference between groups was maintained. The discounting functions based on the last 3 days of training at the shorter delays are illustrated in Figure 7d. Again, there was a main effect of treatment $\left(\mathrm{F}_{(1,60)}=5.40, p=0.024\right)$ and delay $\left(\mathrm{F}_{(4,60)}=\right.$ $31.79, p<0.001)$, but no significant interaction effect $(p=0.86)$, indicating that the adolescent CORT-treated rats made significantly more impulsive choices at all of the delays to large reinforcement. No consistent significant differences were found between groups on any other measures on the task, including reaction time to choose a lever or initiate a trial, or on trial omissions (data not shown).

\section{DISCUSSION}

Chronic CORT exposure specifically during adolescence produces enduring changes in the expression of impulsive behaviors well into adulthood, long after the cessation of CORT treatment. Interestingly, adolescent CORT exposure resulted in bidirectional effects on impulsivity depending on the dimension of impulsive behavior analyzed. Adolescent CORT-treated rats were less impulsive on measures of impulsive action. In particular, these rats were better able to inhibit a prepotent response on the SSRTT. Similarly, adolescent CORT-treated rats were less impulsive at increasing ITIs on the 5CSRTT, suggesting that the treatment either increased their ability to inhibit an ongoing response or wait for the response signal. The results of the SSRTT strongly suggest that adolescent CORT treatment increases the ability to inhibit responding. To provide further support for this conclusion, we also tested adolescent CORT-treated rats on the delay-discounting task, which assesses impulsive choice or the ability to wait for a bigger reward. Unlike the results from the 5CSRTT and SSRTT, the adolescent CORT treatment markedly increased impulsive choice, with the CORT-treated animals selecting the immediate, small reinforcer at a greater frequency than the larger reinforcer even at a very short $3 \mathrm{~s}$ delay. Therefore, adolescent CORT treatment appears to increase 

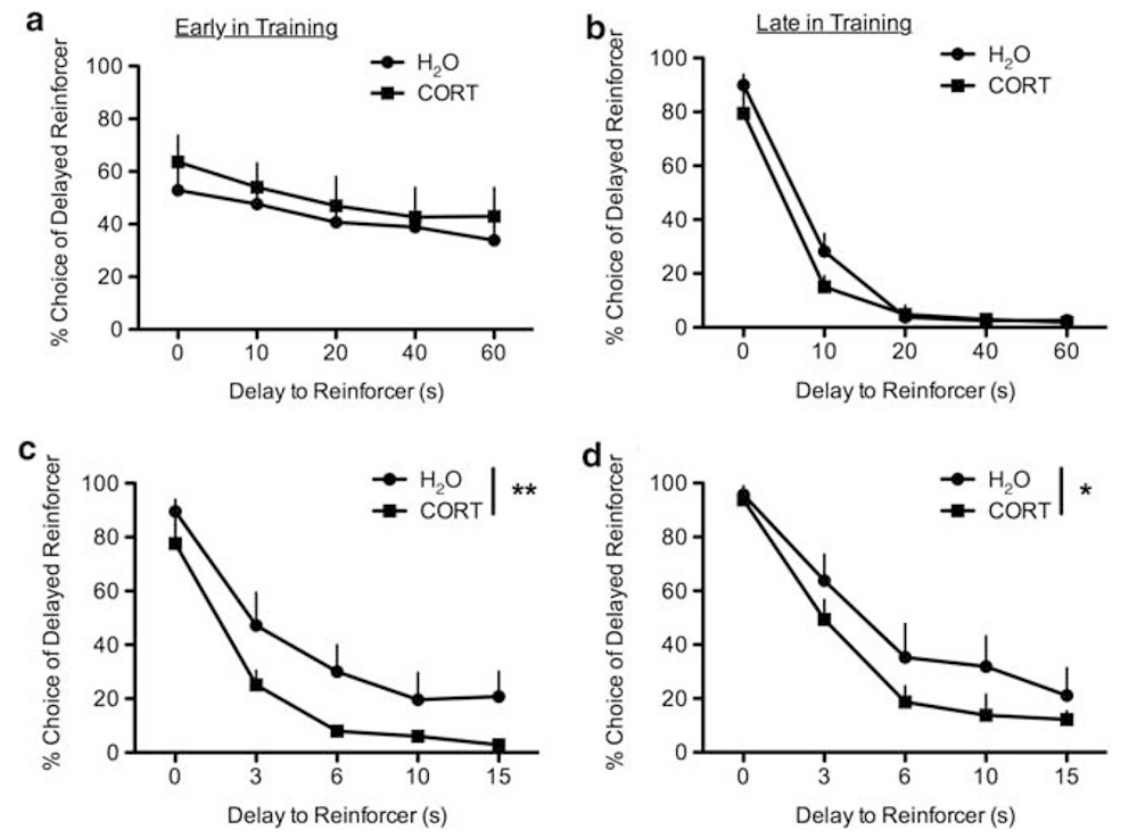

Figure 7 Chronic adolescent corticosterone (CORT) increases impulsive choice on the delay-discounting task. When the range of delays to the larger reinforcer were long (maximum $60 \mathrm{~s}$ ), there was no significant difference between adolescent CORT-treated and control rats on the discounting function either early (a) or late (b) in training. However, when the range of delays to large reinforcement were short (maximum I5s), adolescent CORT treatment caused steeper discounting functions both early (c) and late (d) in training at the new delays, indicating an increased impulsive choice, ${ }^{*} p<0.05,{ }^{*} * P<0.01$.

impulsive choice while simultaneously being able to decrease impulsive action.

Notably, yohimbine caused the adolescent CORT-treated animals to make more impulsive responses on the 5CSRTT. When tested under conditions of the yohimbine challenge, the animals were not asked to adjust their behavior on the basis of a changing ITI, and thus, the yohimbine effect may reflect a decrease in the ability to wait for the stimulus before making a response-conditions under which the adolescent CORT-treated animals were particularly sensitive. In addition, although the increase in yohimbineinduced impulsivity could not be attributed to an increase in baseline levels of phosphorylated CREB, the CORTtreated animals did show an increase in GluR1 expression that was in the active, phosphorylated state. Therefore, upon yohimbine injection, the adolescent CORT-treated animals may have higher levels of AMPA receptor signaling/ trafficking and/or PKA activity that could lead to acute increases in phosphorylated CREB that are not observed at baseline, but may have been observed in response to yohimbine, as was demonstrated by Sun et al (2010). This possible difference in reactivity to norepinephrine-stimulated cAMP activity in the IOFC could also put adolescent CORT-treated animals at risk for behaving impulsively under stressful conditions, though this was not explicitly tested here. Studies of the interaction between stress, norepinephrine, and GluR1 activity in the hippocampus do indicate that norepinephrine and stress can lower the threshold for phosphorylation-dependent LTP-induced incorporation of GluR1 (Hu et al, 2007). If similar mechanisms exist in the IOFC, then the high levels of pGluR1 produced by chronic adolescent CORT could potentiate the effects of noradrenergic activation on impulsivity. In addition, increased PKA activation has been associated with disruption of PFC-dependent tasks, and the increase in
pGluR1 at the PKA site ser845 could indicate an increase in PKA activity that could be exacerbated by yohimbine to produce behavioral disruptions including greater impulsivity (Taylor et al, 1999; Arnsten et al, 2005).

Stress during adolescence has been associated with an increased risk for developing disorders associated with high impulsivity, including substance abuse and other psychiatric disorders (Moeller et al, 2001). Stress exposure during certain vulnerability periods, including adolescence, is known to alter how the HPA axis responds to future stressors (Barha et al, 2011; Burke et al, 2010; Isgor et al, 2004; Weintraub et al, 2010). Therefore, chronic high levels of the hormone corticosterone, which is released by the adrenal glands after HPA axis activation, could produce permanent changes in response to stressors long after the CORT exposure has ended. Indeed, several studies have shown that chronic stress or the presence of an anxiety disorder can increase sensitivity to the effects of yohimbine (Charney et al, 1984; Park et al, 2001; Rosen et al, 1999; Zoladz et al, 2008). However, the majority of differences in impulsivity observed here are unlikely to be explained by altered HPA axis function, but rather by long-lasting neuroplasticity in the circuits that regulate impulsive behavior and decision-making. Indeed, several studies involving adolescent stress exposure report that in adulthood the animals have no difference in baseline CORT levels, though they may have a reduction in peak CORT levels or faster negative feedback after an acute stressor (Weintraub et al, 2010; Burke et al, 2010; Barha et al, 2011).

The ability of chronic adolescent CORT to actually decrease measures of impulsivity on the 5CSRTT and SSRTT could reflect neuroplasticity that results in an increased sensitivity to the punishment that is used in both of these tests when an inappropriate response is made. Future studies could include analysis of physiological 
measures like heart rate during the time out to determine whether rats previously exposed to CORT find the punishment more stressful than controls, and analysis of posterror slowing could assess how they learn after a mistake. Nevertheless, this explanation is supported by the fact that the adolescent CORT-treated animals were more impulsive on the delay-discounting procedure, which does not include a punishment component. Another interpretation of our results is that exposure to high levels of CORT during adolescence remodels brain circuits in a manner that makes individuals better able to cope with stressful situations in the future, and thus better able to inhibit responding under changing conditions. Indeed, several studies have indicated that chronic stress during adolescence can confer resilience to stressful events later in life (Lyons et al, 2010), and the adolescent CORT-treated animals were only less impulsive on tests that involved changing parameters, not when the task was well learned or parameters were stable.

All of these accounts could lead to the results observed in the present studies where animals show reduced impulsive action. The fact that adolescent CORT treatment increases impulsive choice would not be a direct result of any differences in sensitivity to punishment, coping, or anxietylike behavior. Increased impulsive choice, however, could result from an inability to bridge delays between actions and outcomes and/or a greater perceived reduction in the value of a delayed reward. Likewise, the differences in different aspects of impulsivity could represent changes in specific brain circuits induced by chronic CORT exposure. For example, whereas yohimbine-induced impulsivity involves activation of specific signaling cascades in the lOFC (Sun et al, 2010), lesions of the infralimbic region of $\mathrm{PFC}$, not $\mathrm{lOFC}$, have been reported to increase impulsive responding in the face of increasing ITIs on the 5CSRTT (Chudasama et al, 2003). Similarly, behavioral inhibition on the SSRTT may be more dependent on dorsal medial PFC and dorsal striatal activity (Bari et al, 2011; Eagle and Robbins, 2003a; Eagle et al, 2011), whereas choice behavior on the delay-discounting task may be more dependent on a circuit involving the lOFC and ventral striatum (Bezzina et al, 2008; Bezzina et al, 2007; Mobini et al, 2002). Whatever the mechanism, an increase in the likelihood of making impulsive decisions, particularly for immediate 'gratification', could certainly put individuals at a higher risk for developing addictive disorders. It may be this increase in impulsive choice that underlies clinical reports that adolescents exposed to high levels of stress have an increased likelihood to develop addictive disorders (Covault et al, 2007; Lloyd and Turner, 2008; Pilowsky et al, 2009). Moreover, the increase in yohimbine-induced impulsivity represents an additional risk factor for psychiatric disorders, especially addiction. Drug addicts are known to be more likely to relapse after a period of abstinence in response to stress (Sinha, 2007; Stewart, 2003), and acute stress increases the propensity to use drugs (Sinha, 2001). Therefore, although baseline levels of behavioral inhibition may not be impaired after adolescent CORT exposure, the propensity to increase impulsivity in response to statedependent changes could put these individuals at a greater risk for problem drug use.

Although many studies have not attempted to fractionate impulsivity when assessing risk for psychiatric disorders, this is not the first study to find that different measures of impulsivity are not always correlated. Rats identified as having extremely high impulsivity on the 5CSRTT (upper $25 \%$ of population) do not show high novelty reactivity, which is considered another measure of impulsivity or risk taking (Belin et al, 2008; Molander et al, 2011). In humans, children with ADHD who are considered to be more impulsive showed either an increase in impulsive action or impulsive choice, but behavior on the two types of tasks was not correlated (Sonuga-Barke, 2002). In addition, a recent study found that adolescent atomoxetine treatment decreases impulsive choice without affecting impulsive action in adulthood (Sun et al, 2012). Therefore, exposure to high levels of stress hormones during adolescence may manifest as differential risk for certain psychiatric diseases. In addition, different forms of impulsivity may increase risk for developing an addiction to specific substances. For example, high innate impulsivity on the 5CSRTT is associated with the development of compulsive cocaine-, but not heroin-seeking behavior (Belin et al, 2008; Economidou et al, 2009; McNamara et al, 2010). On the other hand, impulsive choice on delay-discounting tasks has been associated with risk for developing an addiction to several drugs of abuse, and drug addiction is often characterized by more impulsive behavior (increased use or relapse) under stressful conditions, suggesting that chronic adolescent stress hormone exposure could certainly put individuals at risk for developing addictive disorders (Jentsch and Taylor, 1999; Moeller et al, 2001; de Wit, 2009). Impulsive choice on delay discounting may be more likely to influence addiction to specific substances, but more research is needed to determine how specific types of impulsivity confer risk for addiction to different substances.

In conclusion, we find that chronic adolescent CORT exposure does not globally alter general cognitive function or attentional ability and actually reduces measures of impulsive action. However, adolescent CORT exposure did significantly increase impulsive choice and impulsive action induced by yohimbine challenge. The behavioral outcomes identified here may be explained by alterations in AMPA receptor/PKA activity in the lateral OFC, but further research is needed to verify this hypothesis. Nevertheless, the results do indicate that individuals exposed to chronic, high levels of stress hormones during adolescence may be at an increased risk for developing psychiatric disorders including addiction because of an increased likelihood to make impulsive choices.

\section{ACKNOWLEDGEMENTS}

We thank Jessica Gordon for her technical assistance. This work was supported by the Interdisciplinary Research Consortium on Stress, Self-control and Addiction (UL1DE19586 (Sinha)) and the NIH Roadmap for Medial Research/Common Fund, PHS AA017537, DA011717, MH066172 and the Connecticut Department of Mental Health and Addiction Services (Taylor).

\section{DISCLOSURE}

The authors declare no conflict of interest. 


\section{REFERENCES}

Andersen SL, Teicher MH (2009). Desperately driven and no brakes: developmental stress exposure and subsequent risk for substance abuse. Neurosci Biobehav Rev 33: 516-524.

Arnsten AF, Ramos BP, Birnbaum SG, Taylor JR (2005). Protein kinase $\mathrm{A}$ as a therapeutic target for memory disorders: rationale and challenges. Trends Mol Med 11: 121-128.

Barha CK, Brummelte S, Lieblich SE, Galea LAM (2011). Chronic restraint stress in adolescence differentially influences hypothalamic-pituitary-adrenal axis function and adult hippocampal neurogenesis in male and female rats. Hippocampus 21: 1216-1227.

Bari A, Mar AC, Theobald DE, Elands SA, Oganya KCNA, Eagle DM et al (2011). Prefrontal and monoaminergic contributions to stop-signal task performance in rats. J Neurosci 31: 9254-9263.

Belin D, Mar AC, Dalley JW, Robbins TW, Everitt BJ (2008). High impulsivity predicts the switch to compulsive cocaine-taking. Science 320: 1352-1355.

Bezzina G, Body S, Cheung THC, Hampson CL, Bradshaw CM, Szabadi E et al (2008). Effect of disconnecting the orbital prefrontal cortex from the nucleus accumbens core on intertemporal choice behaviour: a quantitative analysis. Behav Brain Res 191: 272-279.

Bezzina G, Cheung THC, Asgari K, Hampson CL, Body S, Bradshaw CM et al (2007). Effects of quinolinic acid-induced lesions of the nucleus accumbens core on inter-temporal choice: a quantitative analysis. Psychopharmacol 195: 71-84.

Bourke CH, Neigh GN (2011). Behavioral effects of chronic adolescent stress are sustained and sexually dimorphic. Horm Behav 60: 112-120.

Burke AR, Renner KJ, Forster GL, Watt MJ (2010). Adolescent social defeat alters neural, endocrine and behavioral responses to amphetamine in adult male rats. Brain Res 1352: 147-156.

Chambers RA, Taylor JR, Potenza MN (2003). Developmental neurocircuitry of motivation in adolescence: a critical period of addiction vulnerability. Am J Psychiatry 160: 1041-1052.

Charney DS, Heninger GR, Breier A (1984). Noradrenergic function in panic anxiety. Effects of yohimbine in healthy subjects and patients with agoraphobia and panic disorder. Arch Gen Psychiatry 41: 751-763.

Chudasama Y, Passetti F, Rhodes SEV, Lopian D, Desai A, Robbins TW (2003). Dissociable aspects of performance on the 5-choice serial reaction time task following lesions of the dorsal anterior cingulate, infralimbic and orbitofrontal cortex in the rat: differential effects on selectivity, impulsivity and compulsivity. Behav Brain Res 146: 105-119.

Covault J, Tennen H, Armeli S, Conner TS, Herman AI, Cillessen AHN et al (2007). Interactive effects of the serotonin transporter 5-HTTLPR polymorphism and stressful life events on college student drinking and drug use. Biol Psychiatry 61: 609-616.

de Wit H (2009). Impulsivity as a determinant and consequence of drug use: a review of underlying processes. Addiction Biol 14: 22-31.

Eagle DM, Robbins TW (2003a). Inhibitory control in rats performing a stop-signal reaction-time task: effects of lesions of the medial striatum and d-amphetamine. Behavioral Neurosci 117: 1302-1317.

Eagle DM, Robbins TW (2003b). Lesions of the medial prefrontal cortex or nucleus accumbens core do not impair inhibitory control in rats performing a stop-signal reaction time task. Behav Brain Res 146: 131-144.

Eagle DM, Wong JCK, Allan ME, Mar AC, Theobald DE, Robbins TW (2011). Contrasting roles for dopamine D1 and D2 receptor subtypes in the dorsomedial striatum but not the nucleus accumbens core during behavioral inhibition in the stop-signal task in rats. $J$ Neurosci 31: 7349-7356.

Economidou D, Pelloux Y, Robbins TW, Dalley JW, Everitt BJ (2009). High impulsivity predicts relapse to cocaine-seeking after punishment-induced abstinence. Biol Psychiatry 65: 851-856.

Evenden J (1999). Impulsivity: a discussion of clinical and experimental findings. J Psychopharmacol 13: 180-192.

Fox HC, Bergquist KL, Peihua G, Sinha R (2010). Interactive effects of cumulative stress and impulsivity on alcohol consumption. Alcohol Clin Exp Res 34: 1376-1385.

Gourley SL, Kedves AT, Olausson P, Taylor JR (2009). A history of corticosterone exposure regulates fear extinction and cortical NR2B, GluR2/3, and BDNF. Neuropsychopharmacology 34: 707-716.

Gourley SL, Kiraly DD, Howell JL, Olausson P, Taylor JR (2008a). Acute hippocampal brain-derived neurotrophic factor restores motivational and forced swim performance after corticosterone. Biol Psychiatry 64: 884-890.

Gourley SL, Taylor JR (2009). Recapitulation and reversal of a persistent depression-like syndrome in rodents. Current Prot Neurosci Chapter 9, Unit 9.32.

Gourley SL, Wu FJ, Kiraly DD, Ploski JE, Kedves AT, Duman RS et al (2008b). Regionally specific regulation of ERK MAP kinase in a model of antidepressant-sensitive chronic depression. Biol Psychiatry 63: 353-359.

Hu H, Real E, Takamiya K, Kang MG, Ledoux J, Huganir RL et al (2007). Emotion enhances learning via norepinephrine regulation of AMPA-receptor trafficking. Cell 131: 160-173.

Isgor C, Kabbaj M, Akil H, Watson SJ (2004). Delayed effects of chronic variable stress during peripubertal-juvenile period on hippocampal morphology and on cognitive and stress axis functions in rats. Hippocampus 14: 636-648.

Jacobson-Pick S, Richter-Levin G (2010). Differential impact of juvenile stress and corticosterone in juvenility and in adulthood, in male and female rats. Behav Brain Res 214: 268-276.

Jankord R, Solomon MB, Albertz J, Flak JN, Zhang R, Herman JP (2011). Stress vulnerability during adolescent development in rats. Endocrinology 152: 629-638.

Jentsch JD, Taylor JR (1999). Impulsivity resulting from frontostriatal dysfunction in drug abuse: implications for the control of behavior by reward-related stimuli. Psychopharmacology 146: 373-390.

Jentsch JD, Taylor JR (2003). Sex-related differences in spatial divided attention and motor impulsivity in rats. Behav Neurosci 117: 76-83.

Laucht M, Treutlein J, Blomeyer D, Buchmann AF, Schmid B, Becker $\mathrm{K}$ et al (2009a). Interaction between the 5-HTTLPR serotonin transporter polymorphism and environmental adversity for mood and anxiety psychopathology: evidence from a high-risk community sample of young adults. Int J Neuropsychopharmacol 12: 737-747.

Laucht M, Treutlein J, Schmid B, Blomeyer D, Becker K, Buchmann AF et al (2009b). Impact of psychosocial adversity on alcohol intake in young adults: moderation by the LL genotype of the serotonin transporter polymorphism. Biol Psychiatry 66: 102-109.

Lewis DA (1997). Development of the prefrontal cortex during adolescence: insights into vulnerable neural circuits in schizophrenia. Neuropsychopharmacology 16: 385-398.

Lloyd DA, Turner RJ (2008). Cumulative lifetime adversities and alcohol dependence in adolescence and young adulthood. Drug Alcohol Dep 93: 217-226.

Lyons DM, Lopez JM, Yang C, Schatzberg AF (2000). Stress-level cortisol treatment impairs inhibitory control of behavior in monkeys. J Neurosci 20: 7816-7821.

Lyons DM, Parker KJ, Schatzberg AF (2010). Animal models of early life stress: implications for understanding resilience. Developmental Psychobiol 52: 616-624.

Mar AC, Robbins TW (2007). Delay discounting and impulsive choice in the rat. Cur Prot Neurosci Chapter 8, Unit 8.22.

Mar AC, Walker ALJ, Theobald DE, Eagle DM, Robbins TW (2011). Dissociable effects of lesions to orbitofrontal cortex subregions on impulsive choice in the rat. J Neurosci 31: 6398-6404. 
McNamara R, Dalley JW, Robbins TW, Everitt BJ, Belin D (2010). Trait-like impulsivity does not predict escalation of heroin self-administration in the rat. Psychopharmacology 212: 453-464.

Mobini S, Body S, Ho M-Y, Bradshaw CM, Szabadi E, Deakin JFW et al (2002). Effects of lesions of the orbitofrontal cortex on sensitivity to delayed and probabilistic reinforcement. Psychopharmacology 160: 290-298.

Moeller FG, Barratt ES, Dougherty DM, Schmitz JM, Swann AC (2001). Psychiatric aspects of impulsivity. Am J Psychiatry 158: 1783-1793.

Molander AC, Mar A, Norbury A, Steventon S, Moreno M, Caprioli $\mathrm{D}$ et al (2011). High impulsivity predicting vulnerability to cocaine addiction in rats: some relationship with novelty preference but not novelty reactivity, anxiety or stress. Psychopharmacology 215: 721-731.

Myers EA, Banihashemi L, Rinaman L (2005). The anxiogenic drug yohimbine activates central viscerosensory circuits in rats. J Comp Neurol 492: 426-441.

Park CR, Campbell AM, Diamond DM (2001). Chronic psychosocial stress impairs learning and memory and increases sensitivity to yohimbine in adult rats. Biol Psychiatry 50: 994-1004.

Pilowsky DJ, Keyes KM, Hasin DS (2009). Adverse childhood events and lifetime alcohol dependence. Am J Pub Health 99: 258-263.

Rosen MI, Kosten TR, Kreek MJ (1999). The effects of naltrexone maintenance on the response to yohimbine in healthy volunteers. Biol Psychiatry 45: 1636-1645.

Sinha R (2001). How does stress increase risk of drug abuse and relapse? Psychopharmacology 158: 343-359.

Sinha R (2007). The role of stress in addiction relapse. Cur Psychiatry Rep 9: 388-395.

Sonuga-Barke EJS (2002). Psychological heterogeneity in AD/ HD - a dual pathway model of behaviour and cognition. Behav Brain Res 130: 29-36.

Spear LP (2000). The adolescent brain and age-related behavioral manifestations. Neurosci Biobehav Rev 24: 417-463.
Stewart J (2003). Stress and relapse to drug seeking: studies in laboratory animals shed light on mechanisms and sources of long-term vulnerability. Am J Addictions 12: 1-17.

Sun H, Cocker PJ, Zeeb FD, Winstanley CA (2012). Chronic atomoxetine treatment during adolescence decreases impulsive choice, but not impulsive action, in adult rats and alters markers of synaptic plasticity in the oribitofrontal cortex. Psychopharmacology 219: 285-301.

Sun H, Green TA, Theobald DEH, Birnbaum SG, Graham DL, Zeeb FD et al (2010). Yohimbine increases impulsivity through activation of cAMP response element binding in the orbitofrontal cortex. Biol Psychiatry 67: 649-656.

Swann AC (2010). Mechanisms of impulsivity in bipolar disorder and related illness. Epidemiol Psichiatr Soc 19: 120-130.

Swann AC, Birnbaum D, Jagar AA, Dougherty DM, Moeller FG (2005). Acute yohimbine increases laboratory-measured impulsivity in normal subjects. Biol Psychiatry 57: 1209-1211.

Taylor JR, Birnbaum S, Ubriani R, Arnsten AF (1999). Activation of cAMP-dependent protein kinase $\mathrm{A}$ in prefrontal cortex impairs working memory performance. J Neurosci 19: RC23.

Toth E, Gersner R, Wilf-Yarkoni A, Raizel H, Dar DE, RichterLevin G et al (2008). Age-dependent effects of chronic stress on brain plasticity and depressive behavior. J Neurochem 107: 522-532.

Weintraub A, Singaravelu J, Bhatnagar S (2010). Enduring and sexspecific effects of adolescent social isolation in rats on adult stress reactivity. Brain Res 1343: 83-92.

Winstanley CA (2011). The utility of rat models of impulsivity in developing pharmacotherapies for impulse control disorders. Brit J Pharmacol 164: 1301-1321.

Xu Z, Zhang Y, Hou B, Gao Y, Wu Y, Zhang C (2011). Chronic corticosterone administration from adolescence through early adulthood attenuates depression-like behaviors in mice. $J$ Affective Dis 131: 128-135.

Zoladz PR, Conrad CD, Fleshner M, Diamond DM (2008). Acute episodes of predator exposure in conjunction with chronic social instability as an animal model of post-traumatic stress disorder. Stress 11: 259-281. 